\title{
Enhancement of Electrodynamic Tether Electron Current Collection Using Radio Frequency Power
}

\author{
Éric Choinière ${ }^{1}$, Brian E. Gilchrist ${ }^{2}$ and Sven G. Bilén ${ }^{3}$ \\ ${ }^{I}$ Graduate Student, EECS Department, University of Michigan, Ann Arbor, Michigan 48109 \\ ${ }^{2}$ Associate Professor, EECS \& AOSS Departments, University of Michigan, Ann Arbor, Michigan 48109 \\ ${ }^{3}$ Assistant Professor, CSSL, Pennsylvania State University, University Park, Pennsylvania 16802 \\ (734) 763-5357, echoinie@umich.edu
}

\begin{abstract}
Tether electron current collection in the Orbital Motion Limited regime is one of the limiting factors in power and thrust generation applications of electrodynamic tethers. Injection of radio frequency power along tethers is considered in order to enhance electron current collection. As a basic assessment tool, Particle-In-Cell modeling of the tether system is performed using a 1-d cylindrical code. Comparison of test electron trajectories shows that the time periodic field distribution created by the RF excitation results in electrons being scattered off their usual OML trajectories, which under some conditions increases their probability of being collected by the tether. Analysis of simulation results reveals that large current enhancements can occur at resonance frequencies of the input reactance (where $X_{i n}=0$ ), but at the expense of high RF power. Current enhancement is best measured in terms of the relative current variation per unit of RF power dissipated for every 1-meter section of the tether. Optimum enhancements of about $9 \%$ per RF watt per meter were obtained by simulation at low frequencies $(75 \mathrm{MHz})$. Similar enhancements were observed during experimental measurements on tether samples.
\end{abstract}

\section{INTRODUCTION}

Bare electrodynamic tethers are under consideration for applications such as power and thrust generation for orbiting spacecraft. In these applications, one of the primary concerns is the ability of the system to collect electrons from the surrounding ionospheric plasma in order to maximize the amount of electrical power or thrust provided. For a positive value of the applied DC potential with respect to the surrounding plasma, the amount of electrical power or thrust provided increases with the magnitude of the current flowing on the tether, which in turn is limited by the tether's ability to collect electrons from the surrounding plasma.

Under certain conditions, the current collected by a positively biased long circular cylinder such as a tether immersed in a low-density plasma can be described by the Orbital Motion Limited (OML) current law:

$$
I_{\text {oml }}=2 R \ln e e^{e} \sqrt{2 e V_{p} / m_{e}}
$$

which is based on conservation of angular momentum (Sanmartín, 1999). This relationship is valid provided that the applied voltage is large compared to the electron temperature and that the tether radius is small compared to the Debye length and sheath radius. Thus, it is seen that the amount of current collected per unit length of the tether is controlled by the magnitude of the applied voltage and the radius of the tether.

In order to increase the electron current collection beyond the apparent limitation described above, a technique is proposed here that consists of the injection of a radio frequency signal superposed on the bias potential and propagated along the transmission line formed by the tether sheath structure (Bilén et al., 1995). Analogous to the resonance condition on spherical probes observed by Lepechinsky (1965), a resonance condition on the tether sheath structure was experimentally observed on a long cylindrical probe, which occurred at a frequency slightly under the electron plasma frequency. These experiments consisted in applying a $15-\mathrm{V}$ rms sinusoid voltage onto a bare $10-\mathrm{cm}$

CP552, Space Technology and Applications International Forum-2001, edited by M. S. El-Genk (C) 2001 American Institute of Physics 1-56396-980-7/01/\$18.00 


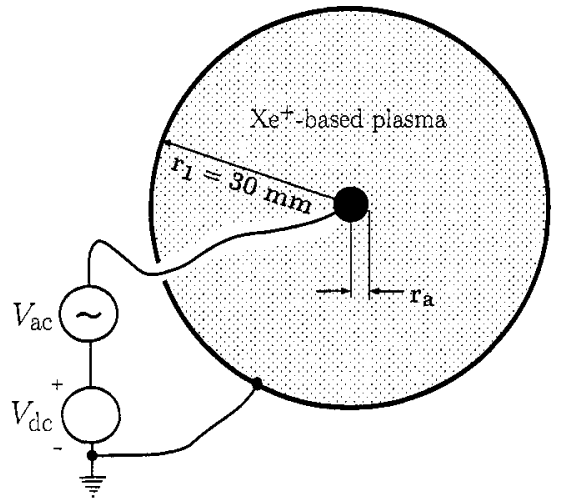

(a) Basic geometry of simplified model

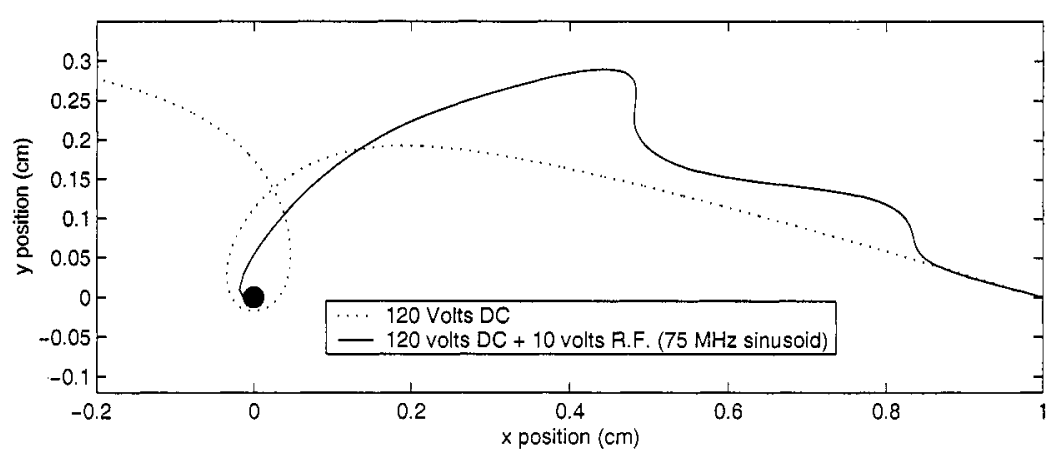

(b) Trajectories of two test electrons through the steady-state, time-periodic potential distributions around a tether for the DC and RF-enhanced cases.

FIGURE 1. Basic PIC Model and RF-Enhancement Principle.

long tungsten tether sample biased at $140-\mathrm{V} \mathrm{DC}$. The simulated tether was immersed in a Xenon plasma at a density of $10^{14} \mathrm{~m}^{-3}$. Results indicated that a large increase in the collected current occurred over a narrow frequency band centered at a frequency slightly under the electron plasma frequency. In the following, we will present the ParticleIn-Cell model that was used, describe the basic physics underlying the possible current enhancement, and finally describe the simulation and experimental results.

\section{PARTICLE-IN-CELL MODEL AND ISSUES}

The Particle-In-Cell (PIC) technique was used in order to model the tether plasma interactions and characterize the enhancement phenomenon that was previously observed experimentally. A 1-dimensional cylindrical implementation of the PIC technique (Verboncoeur, 1993) was adapted and used to this end. The analysis of the simplified problem is based on assumptions of a collisionless plasma, a negligible plasma drift velocity, quasi-static electromagnetic fields, and no external magnetic field. Another important assumption concerns the ion density distribution, which is assumed to be uniform everywhere around the tether; this is the well-known matrix sheath approximation (Lieberman, 1994 and Bilén, 1998) and is valid when $\omega \gg>\omega_{p i}$.

Fig. 1a depicts the geometry of our simplified model. An outer perfect electric conductor at $r=r_{I}$ is used as the boundary condition representing the plasma potential reference, arbitrarily set to zero here. The potential difference between the center and outer conductor is set to a sinusoidal potential variation of amplitude $V_{a c}$ on top of a bias potential $V_{d c}$. It should be noted that the $x p d c l$ computer code (Verboncoeur, 1993) has been modified such that the number of electrons present in the simulation domain remains constant all along the simulation. For this purpose, for every electron collected either on the tether or on the outer conductor, another electron is emitted from the outer conductor with its three velocity components chosen according to a Maxwellian distribution at the bulk plasma electron temperature. As a consequence, the plasma potential is kept constant and very close to zero.

Because of memory and computing power limitations, each individual electron cannot be included in the simulations. Instead, a reduced number of particles with larger charge and mass are simulated. The ratios used for the number of real electrons to the number of simulated particles were in the range of $10^{7}$ to $10^{8}$. In order to compensate for noise in the simulation diagnostics caused by the artificial granularity of the simulation, results were averaged over many $\mathrm{RF}$ cycles after an initial set delay. Many diagnostics were computed using this averaging technique, among which the average collected DC current $\left(I_{d c}\right)$, average sheath width, the fundamental and harmonics of the time-varying current response, the average RF power dissipated in the sheath $\left(P_{r f}\right)$, the input impedance, and the steady-state time periodic electric field distribution. 


\section{BASIC PHYSICS OF THE ENHANCEMENT PHENOMENON}

The known non-linear behavior of the plasma sheath surrounding the tether could be used to obtain an increased DC current with the application of a time-varying potential. An enhancement would be triggered by the time varying electric fields surrounding the tether, which scatter electrons off their OML trajectory by gradually increasing their radially directed velocity, resulting in more incoming electrons being collected on the tether.

From the PIC simulations, an evaluation of the steady-state time periodic electric field distribution (a function of $r$ and $t$ ) was obtained from averages over several RF cycles. This time periodic field is a solution that accounts for all electron plasma dynamics around the tether. Running a test particle (an electron) through this field-solution allows one to predict its trajectory as a function of initial position, velocity, and time of release with respect to the phase of the applied RF signal on the tether.

Fig. $1 \mathrm{~b}$ shows the trajectories of two test electrons with identical initial conditions (thermal velocity corresponding to $T_{e}=0.8 \mathrm{eV}$ and incident angle of 16.6 degrees off the horizontal) through both a DC potential distribution (no RF excitation on the tether) and an RF-enhanced potential distribution. For the case presented here, the initial angular momentum of the electron is too large compared to its radial velocity for it to be collected by the tether with the DC excitation alone. The addition of RF voltage, however, results in the scattering of the electron from the OML trajectory. In the illustrated case, the electron running through the RF-enhanced field is collected. The DC bias was $120 \mathrm{~V}$ in both cases and a $75-\mathrm{MHz}, 10-\mathrm{V}$ amplitude RF signal was added in the second case; the frequency used was lower than the electron plasma frequency of $152 \mathrm{MHz}$ corresponding to an electron plasma density of $2.9 \times 10^{14} \mathrm{~m}^{-3}$.

Although Fig. 1b illustrates a case where the electron running through the RF-enhanced field is collected, this is so only for a certain range of "release phases" measured with respect to the phase of the RF signal source. However, part of the electron population incoming at an incidence angle of 16.6 degrees with the same velocity will be collected, whereas none are in the DC case. An adverse effect is that some of the electrons that would normally be collected under DC conditions (at lower incidence angles, for example) might be scattered off the collection trajectory and be repelled. Although only the collective behavior formed by the combination of all scattered trajectories will determine whether total electron collection is increased or decreased from the DC situation, this simple analysis illustrates how $\mathrm{RF}$ excitation might lead to current enhancement.

\section{SIMULATION AND EXPERIMENTAL RESULTS}

The experimental measurements were performed at the Plasmadynamics and Electric Propulsion Laboratory (PEPL) at University of Michigan, in the Large Vacuum Test Facility (LVTF). A $10-\mathrm{cm}$ long cylindrical tether was immersed in a high-speed Xenon-based plasma generated by a Hall-effect thruster (Haas et al., 1998) developed at University of Michigan and the Air Force. Specific experiment parameters are listed in Table 1. It should be noted that the value used here for $n_{e}$ was obtained by calibrating the model in order to fit the simulated and experimental resonances. This approximation agrees fairly well with that experimentally determined via Langmuir probe and resonance probe measurements. This experiment was performed along with an experiment that examined the DC current collection to bare cylinders in a high speed plasma flow, indicating that the probe used here was collecting in the OML regime with DC bias alone (Gilchrist et al, 2000 and 2001). Also, all simulation results presented here

TABLE 1. Parameters of interest for the experiment.

\begin{tabular}{cc}
\hline Parameter & Values \\
\hline$n_{e}\left(\mathrm{~m}^{-3}\right)$ & $9.4 \times 10^{14}$ \\
$R(\mathrm{~mm})$ & 0.115 \\
$L(\mathrm{~cm})$ & 10 \\
$V_{d c}(\mathrm{~V})$ & 140 \\
$V_{a c}(\mathrm{~V})$ & $21.2(\mathrm{rms}$ value is 15$)$ \\
$T_{e}(\mathrm{eV})$ & $\sim 1-2$ \\
\hline$f_{p e}(\mathrm{MHz})$ & 275 \\
$I_{\text {oml }}(\mathrm{mA})$ & 24.2 \\
\hline
\end{tabular}


were computed using the PIC technique with an $n c 2 p$ (a parameter representing the number of actual particles to simulated particles) of $10^{7}$ and a time step $d t=2.5 \times 10^{-11} \mathrm{~s}$.

Fig. 2 presents a comparison of simulation and experimental results for the average collected current. Both responses show a resonance near $198 \mathrm{MHz}$, where an enhancement of electron collection is observed. In the experiment, an enhancement of approximately $750 \%$ was observed for the DC current. However, the levels of enhancement of simulated and experimental results cannot be compared, because the actual $\mathrm{AC}$ applied voltage on the tether could not be controlled with the experimental configuration that was used. The simulation results, which were realized under conditions of constant input $\mathrm{AC}$ voltage amplitude, show an enhancement of the order of $70 \%$ at the $198 \mathrm{-MHz}$ resonant frequency. Another major distinction between the simulations and measurements is the bandwidth of the enhancement, which was much smaller in the experiment than in the simulations.

The enhancement effect of DC current is associated with an increase of the time-averaged sheath width (shown in Fig. 3a). This increase near the resonance frequency of $198 \mathrm{MHz}$ can be seen even though convergence of the curves has not been obtained due to computing time limitations. The enhancement of the average sheath signifies that the "radius of influence" of the tether on electrons is increased, allowing it to attract a larger number of electrons.

The resonance condition on the DC current collection comes about because the input reactance of the plasmaimmersed tether crosses zero at $f=198 \mathrm{MHz}$, which results in a peak of the injected RF power (see Fig. $3 \mathrm{~b}$ ) since we have an ideal voltage source. In our collisionless model, all RF power is transformed into radially-directed kinetic energy of the local electron plasma, which seems to be the fundamental mechanism leading to the increase of sheath size and the enhancement of electron collection.

Because the RF power level at the tether varies over frequency, simply comparing levels of DC current enhancement for a fixed RF voltage does not yield a useful figure-of-merit. As can be seen in Fig. 3c, the DC current enhancement is proportional to the input $R F$ power for a reasonably low range of power levels. Thus, a useful figureof-merit would be the relative current enhancement per unit watt of $R F$ power per meter; that is, the relative variation of DC current when $1 \mathrm{~W}$ of RF power is injected for every meter of tether. Fig. 3d depicts this figure-of-merit for the simulation results. Results are approximate, because the reference level for the $\mathrm{DC}$ current without $\mathrm{RF}$ excitation has been taken as the $f=50 \mathrm{MHz}$ data, for which a small but non-zero RF power was injected. However, it can clearly be seen from the graph that the optimum current enhancement does not occur at the resonant frequency of $198 \mathrm{MHz}$, which actually presents a local minimum. The "lowest cost" of current enhancement occurs near $f=90 \mathrm{MHz}$, much lower than the resonant frequency; a current enhancement on the order of $9 \%$ is obtained for every watt of RF power injected per meter of tether length. This optimum frequency corresponds to a region of highly non-linear behavior; note that this peak occurs in spite of the low level of injected RF power at this frequency (Fig. 3b). The sharp drop after $f=220 \mathrm{MHz}$ in the relative current enhancement shown on Fig. 3d might explain the dip in the measurement

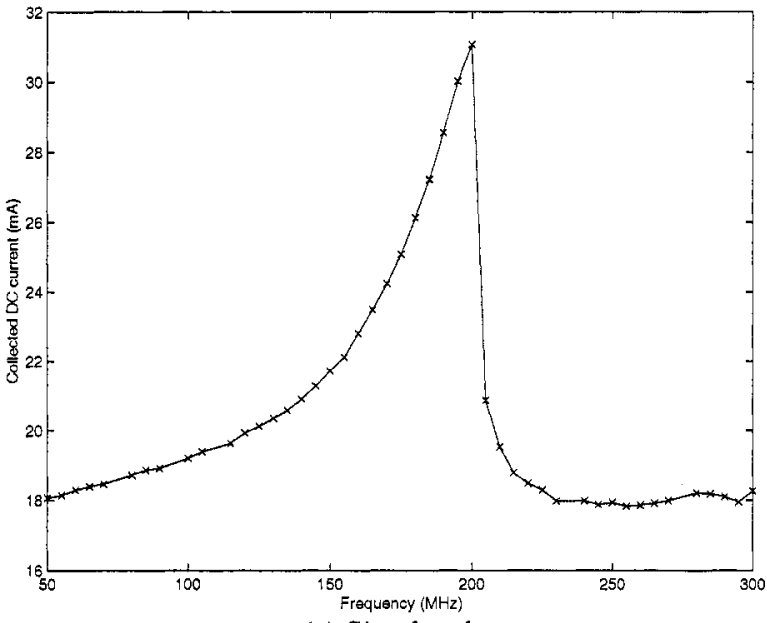

(a) Simulated

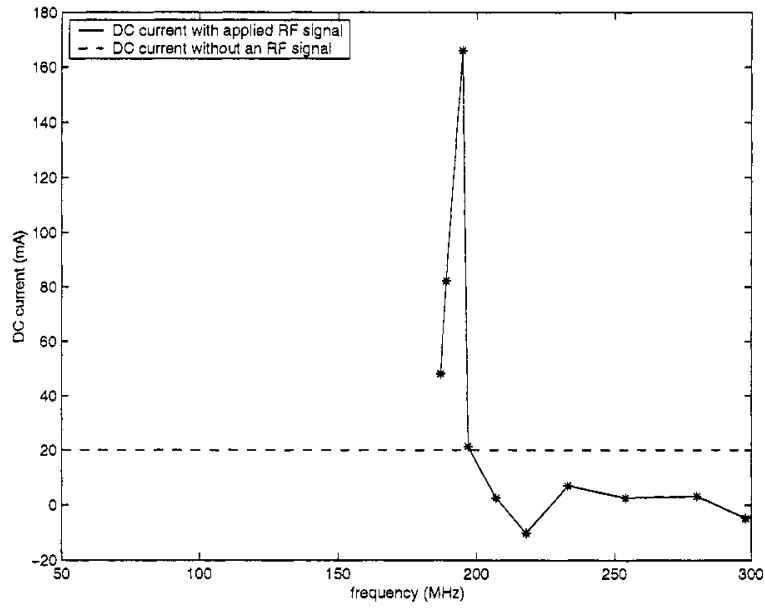

(b) Measured (no data available for $\mathrm{f}<190 \mathrm{MHz}$ )

FIGURE 2. Average Collected Current 


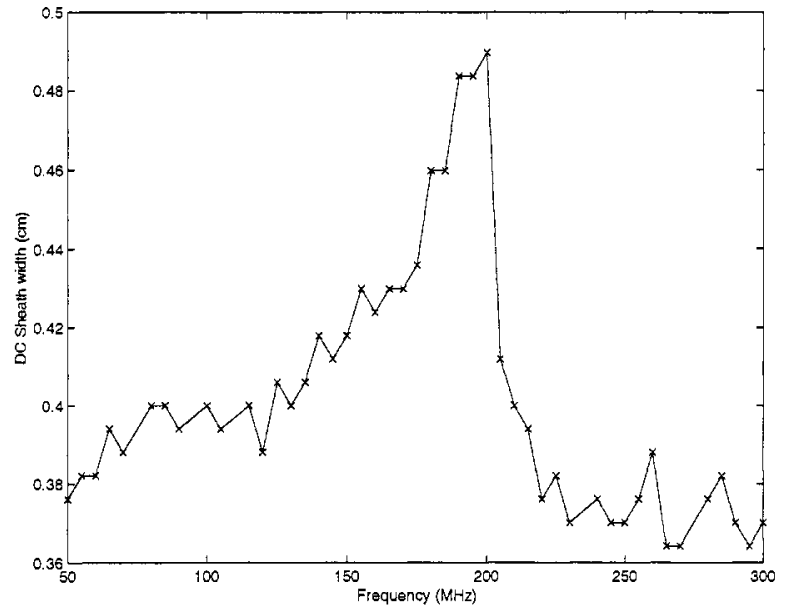

(a) Sheath width

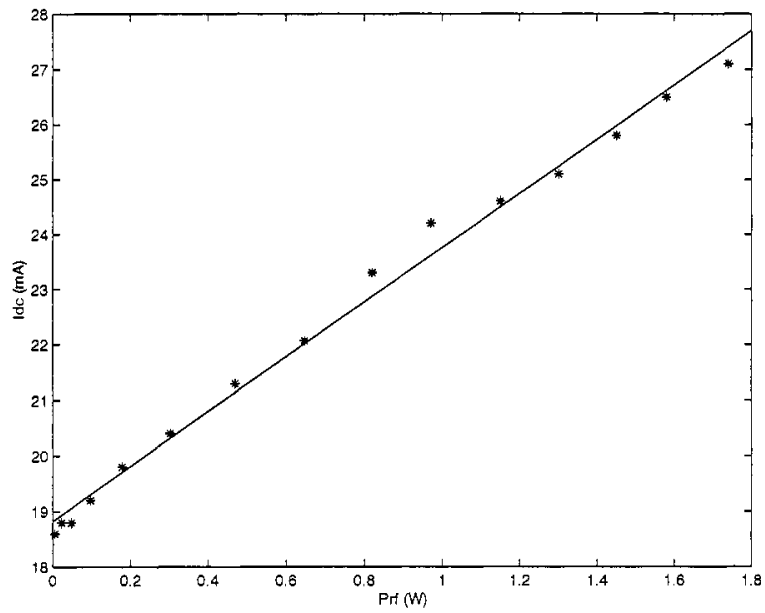

(c) DC collected current vs input RF power (PIC simul.)

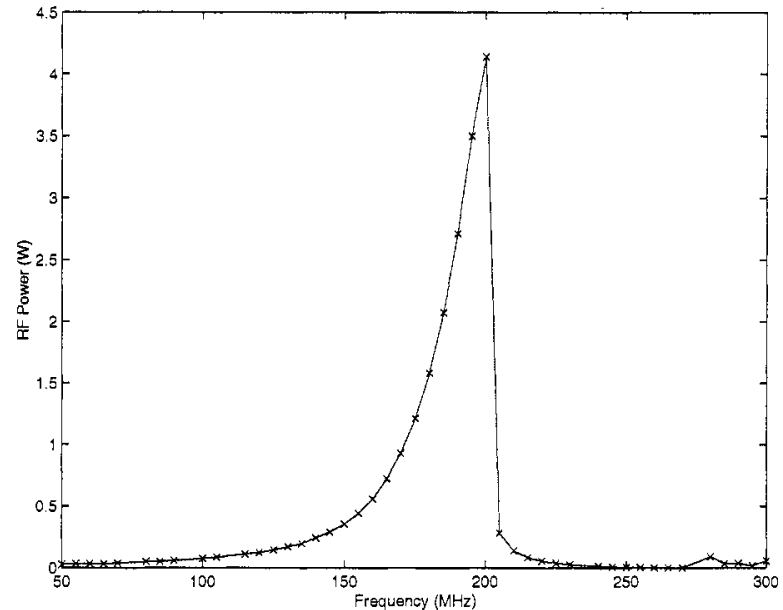

(b) Injected RF power

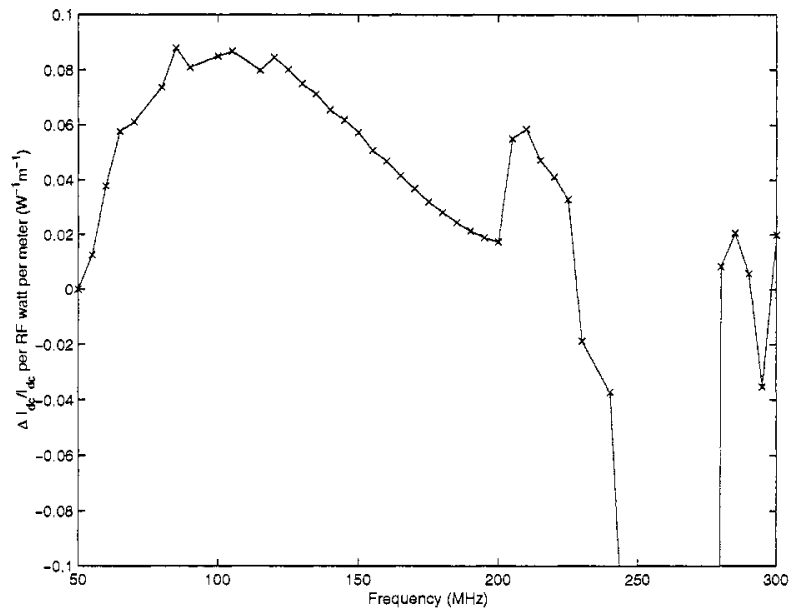

(d) Relative variation of DC current per meter of tether per watt of RF power injected

FIGURE 3. Simulation Diagnostics.

occurring near the same frequency (Fig. 2b), if one assumes that the injected RF power level was approximately constant during the experiment. This is expected since reactance (not shown) is nearly singular around $250 \mathrm{MHz}$.

\section{CONCLUSIONS}

Initial assessment for the injection of radio frequency power on bare tethers as a means of enhancing electron collection in the OML regime was realized using a PIC 1-d cylindrical model and experimental measurements. Large current enhancements were obtained via simulation at resonance frequencies of the input reactance (i.e., where $X_{i n}=0$ ), but at the expense of high RF power. A current enhancement of $750 \%$ was observed experimentally for an unknown input RF power level. Since current enhancement is roughly a linear function of the RF input power level, it is better measured with the relative current variation per unit of RF power along a $1-\mathrm{m}$ section of a tether. Using that figure-of-merit, current enhancements up to $9 \%$ per meter of tether per RF watt were obtained by simulation at frequencies lower than the resonant frequency (near $90 \mathrm{MHz}$ ).

Further investigations should include a comparison of the present simulation results based on the ion-matrix approximation with results obtained when using a Boltzmann distribution for ions based on time-averaged potential. Also, an experiment with controlled power and loss levels should be realized, enabling the direct measurement of the current enhancement figure-of-merit that was presented here. 


\section{NOMENCLATURE}

$n_{e}=$ electron plasma density $\left(\mathrm{m}^{-3}\right)$

$R=$ radius of the tether sample $(\mathrm{m})$

$l=$ length of the tether sample $(\mathrm{m})$

$T_{e}=$ electron temperature $(\mathrm{eV})$

$I_{o m l}=$ collection current predicted by OML theory

$f_{p e}=$ electron plasma frequency $(\mathrm{Hz})$

$P_{r f}=$ RF power (W)

$V_{a c}=\mathrm{AC}$ applied voltage $(\mathrm{V})$

$V_{d c}=\mathrm{DC}$ applied voltage $(\mathrm{V})$

PIC $=$ Particle-In-Cell

OML = Orbital Motion Limit

$e=$ electron charge $(\mathrm{C})$

$m_{e}=$ electron mass $(\mathrm{kg})$

$V_{p}=$ probe voltage $(\mathrm{V})$

$r_{l}=$ radius of simulated outer conductor $(\mathrm{m})$

$r=$ radial distance measured from the center of the tether conductor $(\mathrm{m})$

$t=$ time elapsed, measured from the instant a test particle is released (s)

\section{ACKNOWLEDGMENTS}

The authors would like to thank Prof. A. Gallimore for use of the Large Vacuum Test Facility, the PEPL research group for help in performing the experiments and Dr. Valdis Liepa for the loan of several Radio Frequency sources. Finally, acknowledgments go to the Natural Sciences and Engineering Research Council of Canada as well as the Communications Research Centre Canada, which support this work through scholarships.

\section{REFERENCES}

Bilén, S.G., "Pulse Propagation Along Conductors in Low-Density, Cold Plasmas as Applied to Electrodynamic Tethers in the Ionosphere," Ph. D. thesis, 332 pp., Univ. of Michigan, 1998.

Bilén, S. G., Gilchrist, B. E., Bonifazi, C., and Melchioni, E., "Transient Response of an Electrodynamic Tether System in the Ionosphere: TSS-1 First Results," Radio Science, 1519-1535 (1995).

Gilchrist, B. E., Bilén, Sven G., and Gallimore, Alec D., "Current Collection to Long, Thin Probes in a Dense High-Speed Flowing Plasma," to be presented at the Space Technology \& Applications International Forum 2001.

Gilchrist, B. E., Bilén, Sven G., Patrick, Travis A., and Van Noord, Jonathan L., "Bare Electrodynamic Tether Ground Simulations in a Dense, High-Speed Plasma Flow," AIAA Paper AIAA-2000-3869, 2000.

Haas, James M., Gulczinski III, Frank S., Gallimore, Alec D., Spanjers, Gregory G., and Spores, Ronald A., "Performance Characteristics of a 5 kW Laboratory Hall Thruster," AIAA Paper AIAA-98-3503, 1998.

Lepechinsky, D., "La Sonde à Résonance," L'onde Électrique, 1480-1485 (1965).

Lieberman, Michael A., and Lichtenberg, Allan J., Principles of Plasma Discharges and Materials Processing, John Wiley \& Sons, 1994.

Onishi, T., Martinez-Sanchez, M., and Cooke, D. L., "Computation of Current to a Moving Bare Tether", in Proceedings of the 26th International Electric Propulsion Conference, paper IEPC99-217, 1999.

Sanmartín, J. R., and Estes, R. D., "The Orbital-Motion-Limited Regime of Cylindrical Langmuir Probes," Physics of Plasmas, 395-405 (1999).

Verboncoeur, J. P., Alves, M. V., Vahedi, V., and Birdsall, C. K., "Simultaneous Potential and Circuit Solution for 1D Bounded Plasma Particle Simulation Codes," Journal of Computational Physics, 321-328 (1993). 